\title{
Ivresse et gueule de bois
}

Un Noël à Marseille

From Drunkenness to Hangover. A Christmas in Provence

\section{Jeanne Guien}

\section{(2) OpenEdition}

\section{Journals}

Édition électronique

URL : https://journals.openedition.org/tc/7803

DOI : $10.4000 /$ tc. 7803

ISSN : 1952-420X

Éditeur

Éditions de l'EHESS

\section{Édition imprimée}

Date de publication : 31 octobre 2016

Pagination : 64-73

ISBN : 9782713225291

ISSN : 0248-6016

\section{Référence électronique}

Jeanne Guien, « Ivresse et gueule de bois », Techniques \& Culture [En ligne], 65-66 | 2016, mis en ligne le 31 octobre 2018, consulté le 29 septembre 2022. URL : http://journals.openedition.org/tc/7803 ; DOI : https://doi.org/10.4000/tc.7803 


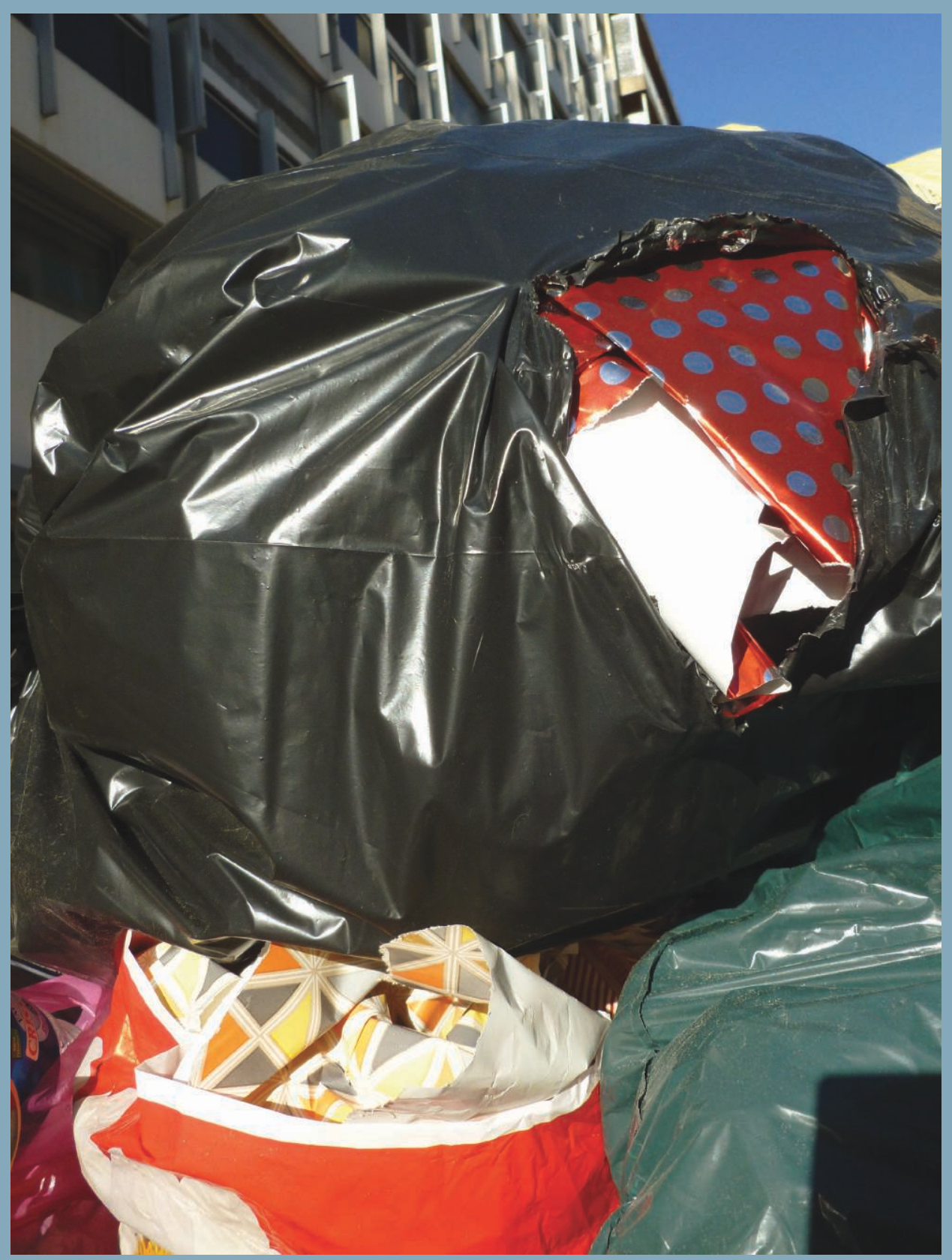




\section{Ivresse et gueule de bois}

\section{Un Noël à Marseille}

En décembre 2010, dans un quartier du 6e arrondissement de Marseille, j'entrepris d'observer les poubelles produites au moment de Noël, cette fête qui, pour une part significative de la population française, occasionne aujourd'hui des échanges et une production de restes exceptionnels. À la façon du rudologue Jean Gouhier, je souhaitais étudier ces restes comme l'« envers » des pratiques de consommation, une consommation festive en l'occurrence. Du 24 au 30 décembre, une à deux fois par jour, je relevais ainsi le contenu des 42 containers à ordures ménagères situés sur mon parcours. En parallèle, je menais des entretiens auprès d'éboueurs et agents de déchetterie du quartier concerné, et observais les pratiques de production de restes dans ma famille et celle d'amies habitant sur le parcours, fêtant Noël et que je visitais plusieurs fois. J'eus aussi des échanges imprévus avec des personnes rencontrées «sur le tas» (éboueurs, biffins, chineurs...) ou avec les passants; car si la fouille des poubelles est une activité solitaire, voire esseulante, elle est aussi provocante et suscite des réactions souvent intéressantes.

Il s'agissait de montrer que l'analyse de la consommation festive doit s'intéresser à des pratiques qui, n'étant pas perçues en elles-mêmes comme festives par les acteurs, en sont souvent exclues, à tort. En effet, «consommer», ce n'est pas seulement ingurgiter (de la nourriture) ou s'approprier (des biens) ; c'est aussi, par exemple, préparer, accompagner, ranger... Gestes censés rester dans l'ombre pour qu'opère la «magie» de la fête, mais qui n'en sont pas moins nécessaires et s'avèrent, à les étudier depuis leurs restes, porteurs de sens. En effet, l'observation systématique des restes suggère que, dans la fête, des choses sont mises pour être détruites, et ne peuvent à ce titre être considérées simplement comme des moyens techniques. Loin de n'être que l'effet accidentel d'actes de consommation abstraits (ingestion ou préhension pures), la production de restes participerait de ce symbolisme social dans lequel la consommation (surtout festive) est toujours prise. Un symbolisme social qui ne se limiterait donc pas à l'usage somptuaire ou agonistique des biens; si la volonté de montrer sa richesse en la distribuant ou la dilapidant joue 
un rôle à Noël, comme le soulignent Mauss dans l'Essai sur le don ou encore Lévi-Strauss dans Les structures élémentaires de la parenté, il y aurait aussi un usage proprement festif du reste, lié à son mode d'être, à ce statut d'être-détruit, d'être-passé, de «reste» compris non seulement comme ce qui «reste de» (a déchu d'un étant extérieur dont il rend possible la production ou la jouissance) mais aussi comme ce qui reste là, fait présence, crée un espace-temps.

Cette interprétation philosophique et anthropologique de nos usages s'autorise du choix d'un échantillon sociologique unifié, l'observation ayant porté sur une population au niveau de vie relativement homogène ${ }^{1}$. Le rythme de cette observation, distinguant un « avant » (préparation), un «pendant» (festoiement) et un «après» (rangement) la fête, a été conservé pour l'exposé, dans la mesure où la variable la plus importante se révéla être le rythme de production de restes, et la manière dont il est constitutif de temps sociaux. Mais, pour cette même raison, il faut aborder cette structure temporelle de manière critique: depuis l'« envers» que sont les restes, c'est une autre narrativité de la fête, une autre temporalité de la jouissance, qui sont apparues, et qui permettent de relire les pratiques.

\section{I'ouverture du bal - déchirures}

J'effectue la première tournée le 24 décembre entre 18 et 19 heures, temps supposé de «préparation » de la fête. J'y observe une production déjà très importante de restes, principalement d'emballages (cartons, papiers, structures de protection plastique...). Emballages de produits alimentaires typiques de Noël (le chapon surgelé Picard et les verrines ont un certain succès cette année-là), mais aussi d'objets manufacturés (cosmétiques, jouets, vêtements...) visiblement destinés à être donnés. Et, déjà, des chutes de papier cadeau - comme si tout avait été déballé, alors même que la fête n'est pas censée avoir commencé.

Si l'on peut supposer que des échanges ont déjà eu lieu (par exemple, le 24 à midi), ces constats suggèrent surtout la spécificité de ces échanges: il s'agit d'offrir, ce qui suppose, de la part de l'offrant, de déballer des choses déjà emballées, pour ensuite les réemballer en vue de les donner à redéballer. Ce, pour les cadeaux (on sort les produits d'un emballage industriel pour en confectionner soi-même un autre) et le repas: à mon passage, mes amies sautent sur l'occasion de me faire descendre deux poubelles, comprenant entre autres des emballages de Blini et sauces à tartiner qui n'ont pas encore été consommés, mais extraits de leur emballage initial, tartinés et disposés sur leurs plats, à leur manière. Ce sont des techniques que l'on pourrait appeler de médiation, transformation d'objet d'échange visant à signaler l'interaction d'une certaine personne dans sa circulation: celle qui donne. Exemple type, le «papier cadeau», qui n'est pas un papier dont on fait cadeau, mais un papier qui fait cadeau, d'abord parce qu'il est fait par le donateur. Ainsi, un cadeau visiblement emballé par le personnel d'un magasin, arborant son logo, peut ne pas procurer le même plaisir. À l'inverse, un simple «mot», une carte ou un dessin, peut «faire cadeau », car ces objets exhibent 
précisément ce qui «compte» dans un cadeau : l'intention, au sens premier de tension, effort vers (quelqu'un), depuis, « de la part de (quelqu'un d'autre).

Il faut voir que cette médiation produit deux types de restes. D'une part, ces emballages soustraits par la préparation, tout de suite jetés, que j'observais dès 19 heures sous forme de déchets. Mais aussi, ces objets ajoutés lors de la préparation, non encore jetés, mais mis pour être ensuite détruits. En effet, à un certain moment et devant un certain public, un bouchon doit «sauter», du papier doit être déchiré, du bolduc, coupé... Destructions qui ne sont jamais, ou jamais seulement, de purs moyens. Si de nombreux gestes techniques semblent partiellement destructeurs, il y a ici comme un surcroît symbolique de destruction: ces objets sont mis pour occasionner ou souligner un geste qui les produit comme restes. L'échange s'accomplit au moyen d'un rituel qui semble presque violent, dans une mise en scène d'autant plus étonnante qu'il s'agit ici de causer le plaisir, ou de signifier que l'on honore un donataire; il semble que, pour le faire accéder à la jouissance d'objets, il faille lui en faire détruire d'autres. Une tension qui peut apparaître, à l'usage, dans certaines hésitations, ou moments conflictuels: peut-on froisser, déchirer, jeter le «petit mot» qui accompagne le cadeau? Le papier cadeau lui-même? Va-t-on les garder? Si oui, combien de temps? Autant d'ambiguités qui suggèrent qu'il existe un certain art, une maîtrise du devenir reste des objets, utiles voire nécessaires au don et à la réception des cadeaux.

La destruction de ces objets permet en effet, d'abord, un dévoilement. Les cadeaux nous paraissent d'autant plus tels, permettent d'autant plus de «faire plaisir» qu'ils sont des «surprises» (autre nom pour cadeau). En témoigne la déception du donateur et du donataire (qui aspire pourtant à savoir, in fine, ce dont il s'agit) si la surprise a «fuité», fuite que l'on qualifiera de «gaffe», erreur et faute morale. De même, l'absence de papier cadeau est perçue comme un manque, auquel il faut pallier (en faisant fermer ses yeux au donataire; en cachant le cadeau derrière son dos; en criant «surprise!»). Notons cependant que, même lorsqu'on sait «à quoi s'attendre» (on sait que la bouteille de champagne contient du champagne), ces pratiques persistent, comme s'il s'agissait avant tout d'isoler la jouissance à venir. Loin de dépendre uniquement de l'étonnement de la surprise ou du plaisir actuel de la prise de possession, celle-ci serait une expérience qu'il faudrait installer dans un cadre à part, dans une durée qui permet de la distinguer des actes de consommation quotidiens, marqués par la prévisibilité et l'ustensilité. En effet, à Noël, le repas est censé être autre chose que la sustentation quotidienne, la «restauration » de nos forces vives; la circulation des objets, autre chose qu'un équipement en biens utiles, « de première nécessité » ou « de consommation courante». D’une quantité ou d'une qualité exceptionnelles, ces échanges doivent aussi être des expériences pleinement vécues, des «moments ». Or l'appropriation ou l'ingestion, et le plaisir qu'ils génèrent, ont tendance à n'être qu'instants éphémères ou flux insaisissables. En faire une date, où la surprise, inconnue mais soupçonnée, est un objet d'attente, dont on peut se «réjouir à l'avance»; faire de l'échange un temps, un «moment à part»; ne sont-ce pas des moyens de conjurer l'éphémérité du plaisir, en en conjurant, par la ritualisation, l'instantanéité et l'obscurité?

Ainsi, au-delà de la qualité ou du prix des biens échangés, la façon dont ils s'échangent joue un rôle clé. Un espace-temps est isolé et ouvert par des techniques qui ne sont pas seulement 
des artifices esthétiques ou des signes de médiation : leur pouvoir hédonique tient avant tout au fait qu'elles permettent d'opérer sur le temps lui-même. C'est parce que le cadeau est toujours un «paquet», et donc (quand bien même on en sait le contenu, «commandé » sur une «liste») une «surprise», que la jouissance possède des seuils, des paliers qui en font une expérience. «Prendre son temps», ouvrir ses cadeaux en plusieurs fois ou avec une lenteur toute cérémonielle, c'est faire honneur au donataire. Poser la bouteille sur la table, ne pas l'ouvrir tout de suite, la laisser être; puis, faire sauter le bouchon, «trinquer», lever son verre... Ce n'est pas encore jouir de «la première gorgée», ou des suivantes; ce sont des avant-goûts sans lesquels la jouissance semble plus frustrante que satisfaisante, car insaisissable. Mais aussi, notons-le, solitaire: user d'objets-seuils, c'est mettre en scène, rendre public et partageable. Même ceux qui ne sont ni donateurs ni donataires, ne jouissant ni du «plaisir d'offrir» ni de la «joie de recevoir », peuvent partager le spectacle du déballage; même ceux qui ne boivent pas trinquent, ou s'adonnent alors à leur chère consommation «occasionnelle». Seul le jaloux, fasciné par la possession du bien échangé, souffre de ne partager «que» ce spectacle; seul le rabat-joie, qui précisément veut gâcher la fête, refuse l'échange et ses techniques. L'art de la fête, qui mobilise ces techniques, est ainsi un art du temps, de la mise en scène d'un plaisir partagé.

Pour comprendre l'usage de ces objets-seuils, leur rôle dans l'organisation des temps sociaux, il faut donc tenir compte du fait que ce moment, cette expérience, est essentiellement fugace, mais aussi irréversible. On ne peut pas, en effet, réoffrir, rejouer la réception d'un cadeau déjà donné. La découverte est irréversible, et c'est en cela qu'elle crée un moment, elle est inaugurale. De fait, les techniques qui la rendent possible présentent cette dimension disruptive: déchirer (du papier), couper (du ruban, du bolduc), «faire péter» ou «sauter» (un bouchon, des pétards ou des feux d'artifice), jeter (des cotillons)... Autant de moyens de commencer une histoire qui ne peut plus qu'avancer, grâce à des objets à détruire, des restes en puissance.

Cette esthétique de l'irréversibilité nous plonge au cœur du problème de la jouissance, cet étrange événement qui doit s'achever pour se réaliser, qui se réalise en s'achevant. Ainsi le suspense de la découverte n'est-il possible, et jouissif, que s'il va vers sa rupture, et l'atteint. S'il «monte» sans jamais se rompre, l'ennui ou l'absurdité nous saisissent; nous voulons son point de rupture, qui est la jouissance même; mais nous le déplorons, car il annonce aussi le déclin. Post coïtum, omne animal triste est ${ }^{2}$-la jouissance laisse place à la tristesse, lorsqu'elle est finie. Mieux: la jouissance est tristesse, parce qu'elle est fin, «fini-tion», pour ainsi dire. Fondamentalement ambivalente, elle est une expérience déchirante, nous divise en deux pôles. Donner au plaisir humain une forme expérimentable, en faire un temps commun au moyen de techniques déchirantes ne nous sauve pas de cette ambivalence, mais permet, en l'accomplissant spectaculairement, rituellement, de vivre pleinement ce dont on ne peut conjurer les limites.

C'est aussi, semble-t-il, à cette fin que naissent les autres restes de Noël. 


\section{La durée des festivités - reliefs}

En effet, la tournée effectuée lors du supposé «temps fort» de la fête (minuit- 1 heure), me donne accès à une autre étape de ce processus. Je suis surprise de ne croiser dans les containers et la rue que quelques chutes éparses de papier cadeau, et des plateaux de fruits de mer vides. De fait, ma mère a insisté pour me faire descendre le nôtre, parce que «ça pue». «Pour le reste, on verra demain » me dit-elle, laissant divers emballages joncher le sol et la table (que l'on ne débarrassait pas). De même, chez mes amies, personne n'émet cette fois l'idée de me faire descendre une poubelle. La fête bat son plein, ses restes sont partout visibles (assiettes et restes de repas sur la table, papiers déchirés au sol), mais personne ne songe à s'en débarrasser. N'est-ce pas précisément parce qu’elle bat son plein?

En effet, des restes ont été produits, mais ne sont pas encore jetés; ils ne sont pas encore des « déchets» (ce dont on se débarrasse, qu'on ne veut plus «chez soi »). À moins de représenter un risque immédiat de salissure (comme le plateau de fruits de mer qui «pue», «coule», "pègue», comme on dit à Marseille), les restes sont d'abord conservés. Non qu'on les recueille, collecte, expose ou stocke pour les «conserver» en un sens muséal; on les laisse simplement être, demeurer là où ils sont tombés après la déchirure. De fait, rassembler des papiers cadeau tout de suite après déballage, aller ramasser un bouchon de champagne à peine ouvert, les jeter... sont plutôt des gestes que l'on condamne comme le propre de celui qui ne sait pas faire la fête, les «réflexes» de celui qui ne se «laisse pas aller»-réintroduit un comportement utilitaire dans un temps censé l'abolir. Les restes commencent, précisément, par rester là; pour que la fête dure et ait lieu, pour que Noël ne s'arrête pas au dernier cadeau ouvert ou à la dernière part de bûche avalée, on use de ce pouvoir qu'a le reste d'ouvrir et de maintenir de la durée.

Aussi le relief, forme spécifique du reste, doit-il être distingué du déchet (res nullius, la chose de personne). Le relief est un «bien», un objet investi de désir et assurant une fonction symbolique. Objet qui a perdu son efficacité technique ou son intégrité physique, il est cependant ce mode sous lequel le reste nous conduit de l'attente au souvenir. Forme floue, vague, il constitue un paysage, conserve un espace-temps qui n'est plus tout à fait l'actualité, qui s'éloigne mais reste encore, comme le « relief» montagneux rappelle au marcheur la contrée qu'il quitte et que bientôt il ne verra plus. Sous cette forme, déformée mais non informe, l'objet n'est ni le désirable, ni le dégoûtant; il est le désiré, ce qui a été désiré, la présence rappelée d'un «trop-vite-passé »; le plaisir s'étiolant et maintenu comme «présent » (autre nom pour cadeau).

Ainsi, la fête peut durer, non pas se succéder et s'achever en instants déchirants; elle acquiert de la continuité. Aussi fête-t-on souvent quelque chose: on s'adonne à la consommation festive lorsqu'il y a une «bonne nouvelle», un «heureux événement» auquel «porter un toast», «trinquer». La consommation ritualisée par les techniques du reste permet de donner corps au plaisir pris si vite (et si seul) lorsqu'il ne tient qu'à un mot.

Les objets reliefs sont donc des éléments nécessaires à la fête, et leur manipulation requiert un art du temps proprement social, qui comprend aussi, par exemple, l'usage de musique, dont la présence immanquable dans les fêtes (y compris celles des moins mélomanes) semble souvent résulter d'un besoin de rythmer, outre les pas, les événements eux-mêmes: de distinguer 
la fête des autres temps, et de distinguer des temps au sein même de la fête. Ainsi une «musique d'ambiance » en marquera-t-elle le début et la fin, une musique «qui bouge» l'apogée: dans cet usage de «fond sonore», la musique permet de mettre en scène nos pratiques. L'usage de divers psychotropes, permettant de se mettre, le temps de la fête, dans un état lui-même à part (souvent qualifié, lui aussi, de "déchiré», « défoncé», «éclaté »), peut aussi être interprété de la sorte: toutes ces techniques font de la fête un temps séparé et homogénéisé, une unité à part mais continue, transcendant les plaisirs éphémères, solitaires et irréversibles qui la parcourent.

Pour cette même raison, il leur faudra aussi conduire à une fin.

\section{Gueule de bois et bonnes résolutions - déchets}

À partir du 25 décembre à 10 heures, j'observe une production de restes massive et particulièrement désordonnée. Leur volume est exceptionnel par rapport au jour précédent et au reste de l'année; entre matin et soir, la physionomie des poubelles aura déjà beaucoup changé; et les éboueurs m’ont confié que leur service peut être doublé ce jour-là. Je constate un certain irrespect des normes en vigueur (heure de sortie de certains containers, règles du tri sélectif, interdiction des décharges sauvages...). Des sacs-poubelles sont descendus à moitié vides; dès le 25 au soir, des calendriers de l'avent, mais aussi des sapins et même un calendrier de l'année 2010 (qui a encore une semaine à vivre) sont jetés. Des techniques de mise au rebut (boîtes ou sacs vides remplies d'autres boîtes, sacs et papiers) sont improvisées pour l'occasion; la production d'emballages est si importante, qu'elle assure une troisième fonction, celle de sac-poubelle.

On observe donc que les restes, après un moment de délai (comme reliefs), deviennent des déchets, sont écartés soudainement et avec désordre. Plutôt que de l'insouciance («pour le reste, on verra demain »), ce désordre semble naître de l'urgence à revenir à la norme, au quotidien. De fait, si ce sont les reliefs qui font durer la fête, l'achever suppose de les écarter; faire des restes des déchets serait la dernière étape. Il s’agirait de revenir à l'ordre, en écartant, conjurant ce qui fait désordre.

Écarter, séparer, permet en effet de (r)établir un ordre; en rangeant, on reconduit le quotidien et on éprouve la norme sociale subvertie par la fête. On «fait le ménage» au sens propre: on fabrique le groupe en séparant désirable et indésirable, propre et impropre, qui devient malpropre. "Nous séparons, nous traçons des frontières, nous rendons visibles les décisions que nous avons prises sur ce que doit être notre foyer, et que nous entendons créer à partir du cadre matériel de la maison», comme le note Mary Douglas (2010 : chap. 4).

Cette reproduction sociale par le nettoyage, véritable « rite de renouvellement», semble d'autant plus nécessaire à Noël où, suite aux échanges de biens, il faut non seulement réassigner à leur place des choses possédées (déplacées) mais aussi définir la place de nouvelles choses (acquises), revoir un inventaire élargi. Il ne s'agit pas seulement d'un rapport (cyclique) entre temps ordinaire et extraordinaires mais aussi d'un rapport (linéaire) entre avant et après. Noël est une fête inaugurale, fin et début d'année d'où l'on mesure le «temps passé», et à venir, par des retrouvailles, 
des bonnes résolutions et des inventaires matériels tout à la fois; une revue des personnes et des biens, de ce «patrimoine» qui les identifie. Aux lendemains de telles fêtes, le foyer est un ensemble modifié, dont la stabilité est en danger, le danger de ces «états de transition» qu'il faut contrôler par une séparation claire, une coupure bien tranchée - coupure que réalise le devenir-déchet.

Ces observations nous reconduisent aussi au problème du plaisir. Le désordre appelle l'ordre autant qu'il s'en écarte dans la mesure où l'ambivalence de la jouissance (toujours prête à se retourner en souffrance) nous empêche de nous y abandonner pleinement - de tomber dans l'excès qui rend les convives malades et les enfants « pourris-gâtés ». Que la gueule de bois succède à l'ivresse -et c'est à peine une image-, c'est aussi la garantie que la licence et l'excès prendront fin, que la passion de l'ordre succédera à celle du désordre. Trier, jeter, débarrasser sont aussi des manières de se purger, décuver, ramasser; de revenir à un ordre dont le bien-fondé ne s'éprouve parfois que négativement, par la subversion. C'est, en tout cas, conjurer matériellement la tentation de l'excès, en assumant les déceptions de la jouissance. Ainsi passe-t-on du désiré à l'indésirable, de l'objet à la chose - l'indéterminé, ce dont « toute identité a disparu » (Douglas 2010: chap. 10). La radicalité de ce changement de statut, de cette déchéance, viendrait en réaction au danger individuel et collectif représenté par le déchet. Déchirer, froisser, mélanger avec d'autres déchets... C'est faire d'objets des matières désormais impossibles à identifier, reconnaître ou récupérer, impossibles à (se) ré-approprier. C'est, paradoxalement, chercher à légitimer le geste qui les écarte: assurer, en détruisant et en achevant les objets, qu'aucun ordre, aucun monde, ne sera possible avec eux. Raisonnement tacite et circulaire qui toucha, en ce Noël 2010, les reliefs de la fête - mais aussi, d'autres objets.

À partir du 26 décembre, j'observe en effet de très nombreux objets récupérables dans les poubelles. Le 26, je rencontre rue Breteuil un premier biffin (ils seront nombreux à arpenter le quartier les jours suivants), occupé à extraire un micro-ondes d'un container. Il récupère avec un fourgon, déjà bien rempli, revend à Bougainville ou porte d'Aix. Des deux containers qu'il fouille, je l'aide à extraire: des vêtements homme et femme, le contenu d'une armoire à pharmacie, une TV, des jouets neufs, un sac... Un chineur croisé le 27 rue Falque n'en finit pas de me décrire ses trouvailles; moi-même je remplis un plein sac à dos, lui-même récupéré. Une quantité importante de biens étrangers à la fête est donc jetée, dans un état tout à fait utilisable. Donnée qui me sembla d'abord une anomalie: des objets qui n'ont été ni objets-seuils ni reliefs, n'ont pas pris part à la fête, viennent aussi jouer le rôle de déchet. Pourquoi?

Comme on l'a dit, Noël s'achève en inventaire et tri; de nouveaux biens sont acquis, il faut leur faire place. De fait, j’observe des objets récupérables jetés dans l'emballage d'un objet semblable plus récent, suggérant que l'on jette parce qu'on a remplacé.

Mais ces interprétations nécessitent une mise en perspective historique. L'habitude de «remplacer» systématiquement ses équipements, de «consommer», épuiser par l'usage, non seulement les «consommables», mais aussi les objets, date du xxe siècle, époque en deçà de laquelle la durabilité des artéfacts était synonyme de fiabilité technique et de valeur culturelle. En ce sens, la part importante de biens «récupérables» dans les poubelles des lendemains de Noël signale un usage et une compréhension spécifiques du reste; spécificité historique dont il faut voir qu'elle peut être décrite à l'aide des différentes catégories qu'on a proposées. 
En effet, l'obsolescence programmée qui caractérise aujourd'hui le cycle de la plupart des produits n'implique pas seulement des procédés techniques (fragilisation des matériaux, dispositifs de panne...) mais aussi des procédés commerciaux: il faut rendre le renouvellement attractif. Des modifications formelles (design) viennent servir un travail de communication (marketing), faisant de la nouveauté un signe valorisateur. Les discours et signes matériels de l'hygiénisme, conseils sanitaires et couleur blanche, purent ainsi être utilisés pour promouvoir le jetable à la salle de bains et à la cuisine; ceux de la légèreté, des matières fines et translucides, faciles à jeter et peu coûteuses à racheter, l'instaurèrent dans les espaces extérieurs et intersticiels, ces temps de pause ou de déplacement où «pratique» devint synonyme de «libre». Les objets-seuils, eux, rendent possible une autre stratégie, consistant à signaler et louer la festivité de produits du quotidien. Antiphrase dont de nombreuses publicités nous invitent à faire notre credo, usant du paradoxe comme d'un acte subversif. Ainsi, Monoprix suggère avec sa campagne « Non au quotidien quotidien» de sublimer nos gestes quotidiens en niant leur quotidienneté, alors même qu'ils ne sont accessibles que sur le mode du rachat systématique. Cette stratégie, qui nous offre, pour lire nos pratiques, le choix entre contradiction et tautologie, promeut l'idée que consommer c'est savoir «craquer» pour des «nouveautés» (un important tabou étant parallèlement jeté sur l'occasion), encensées par des slogans et signalées par des emballages complexes, des «sachets fraîcheur » et autres «boîtes d'origine» dont l'ouverture est même devenue une solennelle pratique ritualisée chez les consommateurs les plus investis, par la production et la mise sur internet de vidéos de unboxing. À « ouverture facile», mais irréversible et à réitérer sans cesse, ces craquages quotidiens permettraient de le «réenchanter», de «faire de chaque jour une fête».

En tant qu'achats d'objets-seuils, de déchets en puissance, ils ont en tous cas pour effet d'accélérer le rythme de la consommation quotidienne, normée par le jeter-racheter et sa temporalité. Une temporalité qui peut entrer en conflit avec celle des rythmes biologiques, avec le besoin d'habitudes structurant la vie matérielle, ou le désir de transmettre les pratiques et de faire culture. Qui fait, en tout cas, disparaître le sens même du « quotidien», de même que celui de la fête. Outre que leur expérience repose sur leur alternance, on ne peut plus en effet penser, comme Bataille dans La part maudite, la consommation festive comme une «consumation»-dépense pure, «usage improductif des ressources»- pour l'opposer à l'« emploi profitable du travail disponible» qui caractérise les mondes du travail et de l'entreprise capitalistes. Car c'est un usage productif de nos forces de consommation qui est fait ici, l'usure et la déchirure des objets par un consommateur quotidiennement fêtard étant un principe fort lucratif de renouvellement du marché.

C'est à ce titre paradoxal que des objets récupérables firent partie de la fête en ce Noël 2010 : traités comme déchets, ils signalent la transformation contemporaine de la consommation en travail, grâce à la promesse de la « fête quotidienne» que deviendrait la vie du consommateur travailleur. Une vie qui n'en finit pas de commencer et de s'achever aussitôt, dans la répétition rapide et indéfinie de gestes rentables et solitaires. 


\section{Notes}

1. Si les quartiers les plus riches de Marseille se trouvent dans les $8^{e}, 7^{e}, 12^{e}$ et $9^{e}$ arrondissements,

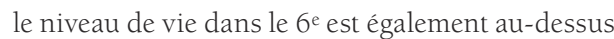
de la moyenne marseillaise. En 2010, les derniers chiffres de l'INSEE (2007) indiquaient un revenu moyen de 17891 euros par UC, pour une moyenne marseillaise de 15284. Le pourcentage d'habitants à bac +3 est double de celui de la ville, tandis que le pourcentage de bénéficiaires de la CMU dans le $6^{\mathrm{e}}$ ne fait que les $2 / 3$ du pourcentage moyen de la ville. Selon un agent de maîtrise du dépôt des éboueurs du $6^{e}$, les déchets de cet arrondissement présentent à Noël les caractéristiques d'un «Noël de riches», à savoir de «durer jusqu'au 2 janvier »: si les déchets des quartiers pauvres sont massifs à Noël, on n'en trouve qu'une fois, contrairement à ceux des quartiers riches, où l'on en trouve moins le 25, mais tout autant les jours suivants, les fêtes ayant le luxe de succéder les unes aux autres.

2. Proverbe latin attribué à Galène ou Galien, dont notre réflexion permet d'ailleurs d'interpréter la suite, moins connue (sive gallus et mulier, «sauf le coq et la femme») comme un déni de jouissance féminine.

\section{I'auteure}

Jeanne Guien est doctorante en philosophie au laboratoire du CETCOPRA à l'université Paris 1 - Panthéon Sorbonne. Pratiquant et organisant la récupération individuellement et collectivement, ses recherches portent sur la réduction de la durée de vie des biens de consommation dans les techniques et les pratiques contemporaines.

\section{Iconographie}

Image d'ouverture. Aux seuils de l'objet. Sur cet exemple de poubelle de Noël, les restes éventrés donnent à lire un parcours type: produit manufacturé à recouvrir avant d'offrir, cadeau emballé pour être redéballé, poubelle biffée par les chasseurs de trésors négligés. (C) Jeanne Guien.

\section{Références}

Bataille, G. 1949 La part maudite. Paris: Éditions de Minuit. Douglas, M. 2010 De la souillure. Paris: Éditions La Découverte.

Gouhier, J. 2000 Au-delà du déchet, le territoire de qualité, Manuel de Rudologie. Rouen: Presses universitaires de Rouen et du Havre.
Lévi-Strauss, C. 1967 Les structures élémentaires de la parenté. Paris: Mouton.

Mauss, M. 2007 [1925] Essai sur le don. Paris: PUF.

\section{Pour citer cet article}

Guien, J. 2016 « Ivresse et gueule de bois - un Noël à Marseille», TechniquesECulture 65-66 «Réparer le monde. Excès, reste et innovation», p. 64-73. 